\title{
INTERNACIONALIZAČ̃̃ E PRÁTICAS DE RESPONSABILIDADE SOCIAL CORPORATIVA EM EMPRESAS BRASILEIRAS
}

Caroline Keidann Soschinski'

Elisane Brandt ${ }^{2}$

Roberto Carlos Klann ${ }^{3}$

\begin{abstract}
Resumo: A divulgação de práticas socialmente responsáveis tem sido requerida cada vez mais por diversos stakeholders, principalmente em mercados desenvolvidos, como o europeu e o norteamericano, tornando-se uma questão estratégica para diversas empresas brasileiras, principalmente aquelas que atuam no mercado externo. Nesse sentido, esta pesquisa objetivou analisar a influência da internacionalização nas práticas de responsabilidade social corporativa (RSC) em empresas brasileiras. A amostra compreendeu 71 empresas durante os anos de 2007 a 2017. Os dados foram analisados por meio de regressão linear múltipla, modelo tobit, com efeitos fixos de setor e ano. Os resultados apontaram que o nível de internacionalização das empresas se mostrou positivamente relacionado à RSC, mas apenas em sua dimensão social e para empresas não reguladas. De forma geral, conclui-se que a busca por ações de RSC por parte das empresas brasileiras tem relação com sua inserção no mercado internacional, como forma de atender possíveis pressões de stakeholders. Dessa forma, contribui-se com a literatura sobre RSC, principalmente a relacionada aos seus determinantes, por evidenciar que não só preocupações sociais e ambientais sustentam tais práticas, mas que decisões estratégicas relacionadas à inserção no mercado externo também podem influenciar tal comportamento.
\end{abstract}

Palavras-chave: Internacionalização. Responsabilidade Social Corporativa. Teoria dos Stakeholders. Responsabilidade Social. Responsabilidade Ambiental.

\footnotetext{
carolinesoschinski@yahoo.com.br - Universidade Regional de Blumenau-SC. Brasil. https://orcid.org/0000-0002-0135-0729

2 elisane_brandt@hotmail.com - Universidade Federal do Paraná-PR. Brasil. https://orcid.org/0000-0001-8129-0908

${ }^{3}$ rklann@furb.br - Universidade Regional de Blumenau-SC. Brasil. https://orcid.org/0000-0002-3498-0938

- DOI: http://dx.doi.org/10.14392/asaa.2019120103

- Artigo submetido em: 21/08/2018. Revisões requeridas: 07/03/2019. Aceito em: 18/03/2019.
} 


\title{
INTERNATIONALIZATION AND PRACTICES OF CORPORATE SOCIAL RESPONSIBILITY IN BRAZILIAN COMPANIES
}

\begin{abstract}
The disclosure of socially responsible practices has been increasingly required by several stakeholders, especially in developed markets such as the European and North American, becoming a strategic issue for several Brazilian companies, especially those that operate in the foreign market. That way, this research aimed to analyze the influence of internationalization on the practices of corporate social responsibility (CSR) in Brazilian companies. The sample comprised 71 companies during the years 2007 to 2017. The data were analyzed through multiple linear regression, tobit model, with fixed effects of sector and year. The results indicate that the level of companies' internationalization was positively related to CSR, but only in its social dimension and for non-regulated companies. In general, it is concluded that the search for CSR actions by the Brazilian companies is related to their insertion in the international market, in order to respond to possible pressures from stakeholders. Therefore, it contributes to the literature on CSR, mainly related to its determinants, noting that not only social and environmental concerns support such practices, but strategic decisions related to the insertion in the external market can also influence such behavior.
\end{abstract}

Keywords: Internationalization. Corporate Social Responsibility. Stakeholder Theory. Social Responsibility. Environmental Responsibility. 


\section{INTRODUÇÃO}

C ada vez mais as empresas se veem pressionadas a divulgar informações com maior transparência sobre seus negócios, dentre estas, estão as informações sobre as práticas de responsabilidade social corporativa (RSC) (Orlitzky, Siegel \& Waldman, 2011). Para Kim e Kim (2010), a RSC é um tema de crescente interesse dos usuários das informações, visto que além de resultados econômicos positivos, espera-se que as empresas também demonstrem suas preocupações com a sociedade, funcionários, acionistas, investidores e demais interessados.

De acordo com Kolk e Van Tulder (2010), é atribuída maior importância ao comportamento socialmente responsável de empresas que são internacionalizadas, o qual decorre da atividade globalizada dessas organizações. Para Detomasi (2008), a necessidade das empresas internacionalizadas em divulgar mais informações é devido ao número de usuários das informações que surgem após o processo de expansão, correspondente aos stakeholders do país de origem e de acolhimento da empresa no exterior.

O processo de internacionalização consiste na expansão da propriedade de uma empresa do país de origem para outro, chamado de país de acolhimento (Mazzioni, 2015). Para Gulamhussen, Pinheiro e Pozzolo (2014), a internacionalização pode ser considerada uma estratégia de crescimento e diversificação das empresas, pois quando estas decidem entrar no mercado estrangeiro buscam criar valor para seus negócios. Assim, a teoria dos stakeholders estabelece uma relação entre a criação de valor e as partes interessadas, pois considera que essa relação é sustentada pelo fato de que as empresas utilizam de seus interessados para criar valor e, em contrapartida, devem reger a boa relação com estes (Freeman, 1994).

Nesse sentido, entende-se que empresas internacionalizadas tendem a se envolver em práticas de RSC para atender a demanda dos seus stakeholders e obter aceitação de suas atividades em contexto internacional. Diversos estudos tem abordado tal problemática, porém, os resultados são em sua maioria divergentes, considerando que algumas pesquisas encontraram relação positiva entre internacionalização e RSC, como Brammer, Pavelin e Porter (2009) no Reino Unido, Kang (2013) e Attig, Boubakri, Ghoul e Guedhami (2016) na América do Norte, Hadjikhani, Lee e Park (2016) na Coreia e China, Agnihotri e Bhattacharya (2016) na Índia, enquanto outras apresentaram relação negativa, como as de Bondy e Starkey (2014) no Reino Unido e Aguilera-Caracuel, Delgado-Márquez e Vidal-Salazar (2014) nos Estados Unidos (EUA).

Um ponto comum nesses estudos é que, em sua maioria, foram realizados em países desenvolvidos. Não há, com exceção do estudo de Agnihotri e Bhattacharya (2016) na Índia, pesquisa sobre o tema com empresas de países em desenvolvimento. Diante disso e da divergência de resultados encontrados na literatura, esta pesquisa procura fornecer evidência no contexto de um país emergente, no caso o Brasil. Assim, tem-se como questão de pesquisa: Qual a influência da internacionalização nas práticas de RSC em empresas brasileiras? Para atender à questão, o objetivo foi de analisar a influência da internacionalização nas práticas de RSC em empresas brasileiras.

A relevância em realizar este estudo em empresas brasileiras se dá em função de que, quando empresas oriundas de países economicamente emergentes se expandem internacionalmente, podem ser vistas com certa desconfiança quanto às suas práticas comerciais. Assim, tendem a aguçar o interesse dos stakeholders, o que implica em maior demanda de informações sobre a conduta empresarial (Agnihotri \& Bhattacharya, 2016). Em contrapartida à essa demanda, as empresas necessitam divulgar informações que reflitam suas preocupações sociais, como forma de perpassar pela distância psíquica existente entre 
países, ou seja, ultrapassar as diferenças geográficas, culturais, econômicas, tecnológicas, regulatórias, do sistema legal e, principalmente, das questões éticas e das práticas de negócios (Campbell, Eden \& Miller, 2012).

A pesquisa de Serra, Albernaz e Ferreira (2007) realizada no Brasil, atenta para o fato de que poucas empresas incluem práticas de RSC em seu planejamento estratégico, contudo, há uma tendência para que essas empresas desenvolvam uma mentalidade socialmente responsável, ao considerar que o processo de expansão internacional, direcionados principalmente para Europa e EUA, exige uma conduta mais sofisticada e consciente sobre tais práticas. Dessa forma, a adaptabilidade dessas empresas às práticas de RSC pode ser um fator decisivo para obter aceitação internacional.

Além disso, evidências na literatura propostas por Abreu, Castro, Soares e Silva Filho (2012) denotam espaço para investigações sobre fatores influenciadores da adesão de práticas socialmente responsáveis. Os autores entendem que as empresas inseridas no contexto brasileiro estão em fase de desenvolvimento e maturação de seu comportamento socialmente responsável. Portanto, esta investigação gera evidências de que a internacionalização pode ser um fator determinante das práticas de RSC.

As contribuições estão voltadas à inclusão de pesquisa empírica no Brasil, por se tratar de um país emergente e em função de que as características dos países podem influenciar as práticas de RSC. Ademais, atende-se à sugestão de Shnayder, Van Rijnsoever e Hekkert (2016), por pesquisas as quais evidenciem os motivos que levam as empresas a adotarem práticas de RSC. Por fim, este estudo fornece evidências sobre as diferenças nas informações de RSC disponibilizadas no contexto brasileiro entre empresas internacionalizadas e locais, em comparação ao observado nos estudos concentrados na América do Norte e Europa.

\section{REVISÃO DE LITERATURA}

\subsection{Teoria dos Stakeholders e Responsabilidade Social Corporativa}

A demanda por informações sobre as práticas empresariais tem aumentado, considerando que os stakeholders não demandam somente informações sobre o desempenho econômico das empresas, mas também sobre questões sociais e ambientais (Orlitzky et al., 2011). Para Kim e Kim (2010), informações sobre o comportamento socialmente responsável é um tema de crescente interesse dos stakeholders.

Os stakeholders consideram uma empresa socialmente responsável quando ela se envolve em questões sociais, desenvolve capacidade de resposta às demandas dos interessados e se mostra responsável para com a sociedade, voluntariamente. De forma prática, destacam-se diversos indicadores os quais podem fornecer indícios sobre um comportamento responsável, como por exemplo, a maneira como a empresa se relaciona com funcionários, a existência de orçamentos e investimentos dedicados à RSC, a comunicação corporativa de relatórios e a relação da empresa com stakeholders (Chapple \& Moon, 2005).

A definição de RSC aparece inicialmente na literatura a partir das investigações de Carroll (1979), o qual destaca a dificuldade em conceituar a RSC por considerar a diversidade de dimensões e contextos que podem englobar um comportamento responsável. Portanto, o autor delineou a RSC como um conjunto de práticas decorrentes de iniciativas das empresas e que podem ser em âmbito econômico, legal e/o discricionário.

Ao tratar sobre as questões que motivam as empresas a se comportarem desta maneira e de forma voluntária, destaca-se a pressão exercida pelos stakeholders. Freeman (1994) é reconhecido na academia por explicar, por um lado, a demanda por informações dos stakeholders e, por outro, a resposta das em- 
presas a essa demanda. O autor afirma que a principal função das organizações é otimizar o bem-estar econômico e o bem-estar social, surgindo, a partir dessa visão, a teoria dos stakeholders.

A teoria dos stakeholders, segundo Freeman (1994), explica a relação entre as organizações e os usuários interessados nas informações. Para o autor, os usuários das informações podem ser definidos por diferentes grupos, como clientes, acionistas, investidores, funcionários, fornecedores, gerentes e administradores, credores, governo e a comunidade. Para Gallego-Álvarez e Ortas (2017) o relacionamento estabelecido entre organizações e stakeholders centra-se na relação entre ética e negócio, sendo que além de atender ao principal objetivo empresarial que é gerar lucro, deve haver a preocupação sobre as demandas sociais e ambientais.

Diante disso, este estudo utiliza a teoria dos stakeholders para apoiar as investigações, ao considerar que as práticas de RSC podem ser percebidas como uma resposta à demanda dos stakeholders, sendo que em empresas internacionalizadas essa pressão é ainda maior.

\subsection{Internacionalização e Responsabilidade Social Corporativa}

A internacionalização é percebida como um fator que motiva as empresas a se engajarem em RSC, o que decorre do fato de serem mais pressionadas pelos stakeholders (Orlitzky et al., 2011). A maior pressão sobre as empresas internacionalizadas é consequência da sua exposição à dualidade de stakeholders, que se referem tanto ao país de origem como de acolhimento (Detomasi, 2008).

Os investimentos em RSC são considerados meios estratégicos das empresas suprirem a necessidade de informações dos stakeholders, visando minimizar efeitos negativos da atividade em âmbito internacional e maximizar a satisfação dos colaboradores (Attig et al., 2016). A decisão de investir em RSC se associa à intenção de proteger a reputação da empresa, reduzir riscos (Kang, 2013), minimizar problemas de comunicação (Zahra, Ireland \& Hitt, 2000) e diminuir efeitos adversos da distância psíquica (Campbell et al., 2012).

Há evidências de investigações sobre a influência da internacionalização na RSC, como o estudo de Attig et al. (2016), o qual investigou o desempenho da RSC sobre o valor da empresa e a internacionalização em empresas dos EUA durante o período de 2002 a 2012. Os resultados mostraram que empresas multinacionais com subsidiárias localizadas em países com determinado ambiente institucional e instituições legais apresentavam mais ações de RSC.

Choi (2017) examinou a relação entre a RSC e a internacionalização em empresas chinesas, durante os anos de 2013 e 2014. Os resultados apresentaram relação positiva entre internacionalização e RSC para as dimensões de gestão ambiental e proteção ao cliente. Agnihotri e Bhattacharya (2016) investigaram como a internacionalização influenciava as práticas de RSC em empresas indianas. Os resultados mostraram que a internacionalização estava positivamente relacionada a RSC e que o aumento de atitudes socialmente responsáveis das empresas internacionalizadas era decorrente da intenção de obter legitimidade.

Por outro lado, Aguilera-Caracuel et al. (2014) investigaram a relação entre internacionalização e RSC em empresas americanas e apontaram que nem todas as dimensões de internacionalização induziam as empresas a melhorarem suas práticas de RSC. Além disso, o estudo constatou outros fatores determinantes das práticas de RSC, como a cultura nacional.

Bondy e Starkey (2014) analisaram a RSC como estratégia adotada pelas empresas internacionalizadas do Reino Unido. Partindo da lógica conceitual, os autores esperavam que à RSC se mostrasse um instrumento chave para as empresas se tornarem competitivas e ganharem espaço no mercado inter- 
nacional, contudo, os resultados demonstraram que as empresas não adotaram práticas de RSC como meio estratégico para a expansão da empresa.

Jung, Lee e Dalbor (2016) investigaram se havia efeito sinérgico da internacionalização e da RSC no desempenho de valor de empresas americanas, durante o período de 2000 a 2011. Os resultados sugeriram que para as empresas que se expandiram internacionalmente e aderiram práticas socialmente responsáveis em conjunto, houve um efeito negativo no desempenho do valor das empresas. Os autores recomendaram aos gestores investigar com precisão o conteúdo das práticas de RSC que pretendem aderir ao expandirem seus negócios em mercados internacionais.

No contexto brasileiro, pode-se citar o estudo de caso realizado por Serra et al. (2007), que analisaram a importância da RSC para o crescimento e internacionalização da empresa Natura Cosméticos S.A. Os autores constataram que a RSC era caracterizada como uma forma de materializar vantagens competitivas e expandir a atuação internacionalmente. De maneira geral, sugeriram que empresas brasileiras precisam se conscientizar sobre atitudes socialmente responsáveis como uma maneira de garantir sua aceitação no exterior.

Com base nos preceitos teóricos apresentados, entende-se que empresas que se expandem internacionalmente são mais propensas a se engajar em RSC, como forma de minimizar os efeitos adversos da distância psíquica existente entre país de origem e de acolhimento. Considera-se que as práticas de RSC podem ser consideradas um aspecto estratégico motivado pelo nível de internacionalização das empresas. Portanto, assume-se $\mathrm{H} 1$ :

H1 - A internacionalização está positivamente relacionada às práticas de RSC em empresas brasileiras.

\section{METODOLOGIA}

A população de pesquisa contempla as empresas públicas listadas na Thomson Reuters Eikon, localizadas no Brasil. Para delimitar a amostra, foram excluídas as empresas que não continham dados para cálculo das variáveis, as empresas financeiras por possuírem características particulares e os outliers, por estarem três desvios padrões distantes da média.

O Painel A da Tabela 1 apresenta as 71 empresas da amostra e suas repetições durante o período, o que totalizou 530 observações. No Painel B, apresentam-se as observações por ano e por tipo de empresa (se internacionalizada ou não), no Painel C, as empresas distribuídas por setores e no Painel D, as empresas que possuíam ADR durante os anos de análise.

Tabela 1 - Amostra da pesquisa

\begin{tabular}{|l|c|c|c|c|c|c|}
\hline \multicolumn{4}{|c|}{ Painel A } & \multicolumn{5}{c|}{ Painel B } \\
\hline Empresas & Repetições & Observações & Ano & Sem internac. & Com internac. & Total \\
\hline 2 & 2 & 4 & 2007 & 1 & 2 & 3 \\
\hline 2 & 3 & 6 & 2008 & 9 & 2 & 11 \\
\hline 9 & 5 & 45 & 2009 & 17 & 5 & 22 \\
\hline 6 & 6 & 36 & 2010 & 42 & 11 & 53 \\
\hline 8 & 7 & 56 & 2011 & 48 & 12 & 60 \\
\hline 25 & 8 & 200 & 2012 & 46 & 15 & 61 \\
\hline 10 & 9 & 90 & 2013 & 48 & 15 & 63 \\
\hline 6 & 10 & 60 & 2014 & 50 & 13 & 63 \\
\hline 3 & 11 & 33 & 2015 & 50 & 14 & 64 \\
\hline
\end{tabular}




\begin{tabular}{|c|c|c|c|c|c|}
\hline \multirow{3}{*}{71} & \multirow{3}{*}{530} & 2016 & 49 & 16 & 65 \\
\hline & & 2017 & 53 & 12 & 65 \\
\hline & & Total & 413 & 117 & 530 \\
\hline \multicolumn{6}{|c|}{ Painel C } \\
\hline \multicolumn{5}{|c|}{ Setores - NAICS Sector Name } & Empresas \\
\hline \multicolumn{5}{|c|}{ Agricultura, Silvicultura, Pesca e Caça } & 1 \\
\hline \multicolumn{5}{|c|}{ Comércio Atacadista } & 1 \\
\hline \multicolumn{5}{|c|}{ Comércio Varejista } & 6 \\
\hline \multicolumn{5}{|c|}{ Construção } & 6 \\
\hline \multicolumn{5}{|c|}{ Cuidados de Saúde e Assistência Social } & 2 \\
\hline \multicolumn{5}{|c|}{ Informação } & 4 \\
\hline \multicolumn{5}{|c|}{ Manufatura } & 20 \\
\hline \multicolumn{5}{|c|}{ Imóveis e Locação } & 4 \\
\hline \multicolumn{5}{|c|}{ Mineração, Pedreiras e Extração de Petróleo e Gás } & 4 \\
\hline \multicolumn{5}{|c|}{ Serviços de Utilidade Pública } & 14 \\
\hline \multicolumn{5}{|c|}{ Serviços Educacionais } & 2 \\
\hline \multicolumn{5}{|c|}{ Serviços Profissionais, Científicos e Técnicos } & 1 \\
\hline \multicolumn{5}{|c|}{ Transporte e Armazenagem } & 6 \\
\hline \multicolumn{5}{|c|}{ Total } & 71 \\
\hline \multicolumn{6}{|c|}{ Painel D } \\
\hline \multicolumn{5}{|c|}{ Empresas com ADR } & 18 \\
\hline
\end{tabular}

A partir da análise principal realizada com a amostra de 71 empresas (530 observações), foram realizados alguns testes de sensibilidade/robustez, a fim de verificar se os resultados sobre a influência da internacionalização na RSC não poderiam estar sendo enviesados por fatores regulatórios, de negociação em outra bolsa de valores ou pela repetição de empresas nos primeiros anos analisados.

Primeiramente, as empresas foram separadas em reguladas e não reguladas. Considerou-se como setor regulado o de telecomunicação (regulado pela Agência Nacional de Telecomunicação - ANATEL), de energia elétrica (regulado pela Agência Nacional de Energia Elétrica - ANEEL) e de petróleo e gás natural (regulado pela Agência Nacional do Petróleo, Gás Natural e Biocombustíveis - ANP). A realização deste teste teve como intuito controlar a regulação de determinados setores como fator passível de influenciar nas práticas de RSC, uma vez que a RSC pode ser motivada por questões regulatórias desses setores e não pela internacionalização de fato.

Posteriormente, testou-se a influência de empresas com ADR (American Depositary Receipt) na Bolsa de Valores de Nova York (NYSE) e internacionalizadas, nas práticas de RSC. A informação sobre a listagem das empresas brasileiras na NYSE foi coletada na base de dados da Economática. Esse teste objetivou verificar se o fato de as empresas possuírem ADR em uma bolsa de valores estrangeira pode ser um fator de influência das práticas de RSC, pois empresas que decidem negociar suas ações fora do país podem estar expostas a demanda de informações dos investidores e acionistas estrangeiros, o que assim como o nível de internacionalização, poderia motivar os investimentos em RSC.

Por fim, como último teste de sensibilidade/robustez, operacionalizou-se o modelo principal somente com as observações de 2010 a 2017, ou seja, foram excluídos os três primeiros anos (2007, 2008 e 2009), para corrigir um possível viés ocasionado pela repetição de empresas nesses primeiros períodos.

As informações referentes às variáveis dependentes e de controle foram coletadas no banco de dados Thomson Reuters Eikon, enquanto que a internacionalização foi coletada no website da Fundação Dom Cabral (FDC), por meio do Relatório Anual de Transnacionalização, disponível desde o ano de 2006. 
Contudo, o ano de 2006 não foi incluído na amostra pois o cálculo de internacionalização deste relatório não foi realizado de acordo com o indicado pela UNCTAD (1995). Portanto, o período deste estudo foi determinado de 2007 até 2017. As variáveis da pesquisa estão dispostas na Tabela 2.

Tabela 2 - Variáveis da pesquisa

\begin{tabular}{|c|c|c|c|c|c|}
\hline \multicolumn{3}{|c|}{ Variáveis dependentes } & Descrição & Cálculo & Autores \\
\hline \multirow{7}{*}{ 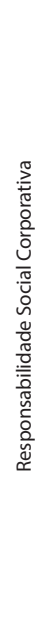 } & \multirow{3}{*}{ 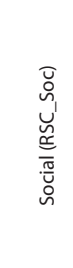 } & Comunidade & $\begin{array}{c}\text { Mede o compromisso da empresa em } \\
\text { proteger a saúde pública e respeitar a } \\
\text { ética empresarial. }\end{array}$ & \multirow{6}{*}{$\begin{array}{l}\text { Pontuação gerada } \\
\text { pe la Th om s o n } \\
\text { Reuters Eikon: } \\
\text { Varia de } 0 \text { a } 100 .\end{array}$} & \multirow{6}{*}{$\begin{array}{l}\text { Jung et al. (2016); Attig et al. } \\
\qquad(2016)\end{array}$} \\
\hline & & Direitos Humanos & $\begin{array}{l}\text { Mede a eficácia da empresa em respeitar } \\
\text { as convenções de direitos humanos. }\end{array}$ & & \\
\hline & & Força de Trabalho & $\begin{array}{l}\text { Mede a eficácia da empresa em manter } \\
\text { o local de trabalho saudável e seguro, } \\
\text { em gerir a satisfação dos empregados } \\
\text { e manter a diversidade e a igualdade de } \\
\text { oportunidades de crescimento. }\end{array}$ & & \\
\hline & \multirow{3}{*}{ 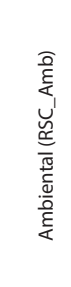 } & Uso de Recursos & $\begin{array}{l}\text { Mede a capacidade da empresa em } \\
\text { reduzir o uso de materiais, de energia e } \\
\text { água e encontrar soluções eco-eficientes. }\end{array}$ & & \\
\hline & & Emissão de poluentes & $\begin{array}{c}\text { Mede a eficácia da empresa em reduzir } \\
\text { a emissão de poluentes nos processos } \\
\text { de produção. }\end{array}$ & & \\
\hline & & & $\begin{array}{c}\text { Mede a capacidade da empresa em } \\
\text { reduzir os custos e encargos ambientais } \\
\text { para seus clientes e criar oportunidades } \\
\text { de mercado através de novas tecnologias } \\
\text { e produtos eco-projetados. }\end{array}$ & & \\
\hline & \multicolumn{2}{|c|}{ Geral (RSC) } & $\begin{array}{l}\text { Mede a RSC pela média das dimensões } \\
\text { social e ambiental. }\end{array}$ & $\underset{/ 2}{\text { RSC_Soc }+ \text { RSC_Amb }}$ & Sugerido pelos autores \\
\hline \multicolumn{3}{|c|}{ Variável independente } & Descrição & Cálculo & Autores \\
\hline \multicolumn{3}{|c|}{ Internacionalização (INTER) } & $\begin{array}{l}\text { Mede o grau de internacionalização de } \\
\text { cada empresa. }\end{array}$ & $\begin{array}{c}(\mathrm{V} \text { Ext/VT })+ \\
(\mathrm{AEext} / \mathrm{AT})+(\mathrm{EExt} / \\
\mathrm{ET}) / 3\end{array}$ & $\begin{array}{l}\text { Bondy e Starkey (2014); } \\
\text { Agnihotri e Bhattacharya } \\
\text { (2016); Hadjikhani et al. } \\
\text { (2016) }\end{array}$ \\
\hline \multicolumn{3}{|c|}{ Variáveis de controle } & Descrição & Cálculo & Autores \\
\hline \multicolumn{3}{|c|}{ Tamanho (TAM) } & $\begin{array}{c}\text { Mede o tamanho da empresa em termos } \\
\text { de ativo total. }\end{array}$ & $\begin{array}{l}\text { Logaritmo Natural } \\
\text { do Ativo Total }\end{array}$ & $\begin{array}{l}\text { Attig et al. (2016); Agnihotri } \\
\text { e Bhattacharya (2016); Ali, } \\
\text { Frynas e Mahmood (2017) }\end{array}$ \\
\hline \multirow{2}{*}{ 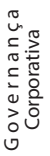 } & \multicolumn{2}{|c|}{ Gestão (GC_Ges) } & $\begin{array}{l}\text { Mede a eficácia da empresa em seguir } \\
\text { as melhores práticas de governança } \\
\text { corporativa. }\end{array}$ & \multirow{2}{*}{$\begin{array}{l}\text { Pontuação gerada } \\
\text { pela Th om s o n } \\
\text { Reuters Eikon: Varia } \\
\text { de } 0 \text { a } 100 .\end{array}$} & \multirow{2}{*}{ Ali et al. (2017) } \\
\hline & \multicolumn{2}{|c|}{ Acionista (GC_Aci) } & $\begin{array}{l}\text { Avalia a eficácia de uma empresa para a } \\
\text { igualdade de tratamento dos acionistas e } \\
\text { o uso de dispositivos anti-takeover. }\end{array}$ & & \\
\hline \multicolumn{3}{|c|}{ Rentabilidade } & Mede a rentabilidade sobre o ativo (ROA) & EBIT / Ativo Total & $\begin{array}{l}\text { Attig et al. (2016); Ali et al. } \\
\text { (2017) }\end{array}$ \\
\hline
\end{tabular}

Nota: VExt: Vendas no exterior. VT: Vendas totais. AEext: Ativo no exterior. AT: Ativo total. EExt: Empregados no exterior. ET: Empregados totais. LN: Logaritmo natural.

Fonte: Dados da pesquisa (2018)

Com base na Tabela 2, ressalta-se alguns detalhes pertinentes acerca das variáveis. Observa-se que a RSC é medida por duas dimensões, uma reflete questões sociais (RSC_Soc) sobre comunidade, direitos humanos e força de trabalho, enquanto a outra reflete as questões ambientais (RSC_Amb), sobre o uso de recursos, emissão de poluentes e inovação ambiental. Essas variáveis foram formuladas por meio de uma metodologia própria da Thomson Reuters Eikon, a qual considera alguns itens como "verdadeiro" ou "falso", outros por valores monetários e outros por volumes ou quantidades físicas. Por meio destes, gera um score final que pode variar de 0 a 100, indicando o score de RSC de cada empresa.

A dimensão social é composta pela média de três scores sobre à comunidade, direitos humanos e força de trabalho. O score voltado para as questões comunitárias é composto por 28 itens que tratam se a empresa tem ou não: política justa de concorrência; política contra suborno e corrupção; política que visa a ética empresarial; política de envolvimento comunitário, entre outros. Além disso, existem itens que envolvem valores monetários, como o total de valor destinado a doações. Assim, o banco de 
dados considera esses 28 itens e formula um score geral para medir o compromisso da empresa com a comunidade.

O score relacionado aos direitos humanos é composto por 11 itens que tratam se a empresa tem ou não: política de direitos humanos; política de trabalho infantil; política de trabalho forçado; iniciativa de comércio ético, entre outros. O score relacionado à força do trabalho é composto por 48 itens que verificam se a empresa tem ou não: política de saúde e segurança; política de saúde e segurança dos colaboradores; política de saúde e segurança da cadeia de suprimentos; política de treinamento e desenvolvimento; política de treinamento de habilidades, entre outros. Para esta dimensão, há itens que medem questões como o índice de rotatividade de empregados, de mulheres no grupo de empregados, entre outros.

A dimensão ambiental é composta pela média de três scores sobre questões voltadas ao uso de recursos, à emissão de poluentes e à inovação ambiental. O score direcionado ao uso de recursos contempla 31 itens que verificam se a empresa tem ou não: política de redução de recursos; política de eficiência da água; política de eficiência energética; política de embalagem sustentável; política ambiental da cadeia de suprimentos; metas de redução de recursos, entre outro. Ademais, envolve valores monetários, como o valor total que a empresa desembolsou em uso de energia.

O score relacionado à emissão de poluentes envolve 40 itens, se a empresa tem ou não: política de emissão; metas de emissões; o total emitido em $\mathrm{CO}_{2}$; compensação de crédito de carbono, se visa a redução de partículas, entre outros. Por fim, o score que mede a inovação ambiental considera 24 itens que verificam se a empresa tem ou não: produtos ambientais; produtos eco projetados; redução de ruído; veículos híbridos, produtos de mínimo impacto; o valor investido pela empresa em pesquisa e desenvolvimento ambiental, entre outros.

Mediante esses 6 scores gerados pelo banco de dados sobre questões sociais e ambientais, calculou-se a média entre os indicadores sociais (comunidade, direitos humanos e força de trabalho), chegando-se ao indicador da dimensão social (RSC_Soc) e a média entre os indicadores ambientais (uso de recursos, emissão de poluentes e inovação ambiental), chegando-se ao indicador da dimensão ambiental (RSC_Amb). Por fim, foi calculada a média entre esses dois indicadores (social e ambiental) para formar uma dimensão geral de RSC (RSC_M).

Com relação à variável de internacionalização, obteve-se acesso aos Relatórios de Transnacionalização elaborados anualmente pela Fundação Dom Cabral, disponíveis no website e elaborados com base no cálculo proposto pela UNCTAD (1995), o qual considera a relação entre os ativos no exterior e ativos totais, a relação entre as vendas no exterior e as vendas totais e a relação entre os funcionários que exercem suas funções no exterior e o total de funcionários. Assim, foi possível medir o grau de internacionalização de cada empresa, que pode variar de 0 a 1, de acordo com a proporção de ativo, vendas e empregados no exterior. Portanto, quanto mais a empresa for internacionalizada, mais próximo de 1 será o seu grau de internacionalização.

No que concerne às variáveis de controle, foram utilizadas o tamanho, a governança corporativa (GC) e a rentabilidade sobre o ativo. As medidas de GC, tanto a direcionada à gestão como aos acionistas, estão disponíveis na Thomson Reuters Eikon e seguem a mesma metodologia de cálculo da RSC, ou seja, podem variar de 0 a 100, dependendo da quantidade de itens atendidos pelas empresas. Existem 56 itens que compõem a GC voltada à gestão (GC_Ges), que mede questões sobre se a empresa tem ou não: política de funções do conselho; comitê de GC; comitê de auditoria; política de independência do conselho; política de diversidade do conselho, entre outros. A variável de GC destinada aos acionistas 
(GC_Aci) é composta por 35 itens sobre se a empresa tem ou não: política de direito dos acionistas; política de direito igualitário de voto; política de engajamento dos acionistas, entre outros.

De acordo com a revisão da literatura de Ali et al. (2017), o tamanho das organizações, a rentabilidade sobre o ativo e os níveis de GC são fatores determinantes das práticas de RSC. Para os autores, empresas maiores e mais rentáveis estão mais expostas a pressão dos stakeholders e possuem mais recursos, o que as instiga a se engajarem com maior intensidade em RSC. De forma semelhante, os autores elencam que organizações que contém melhores práticas de GC também estão positivamente relacionadas as práticas socialmente responsáveis.

Para testar a hipótese da pesquisa, utilizou-se de regressão linear múltipla, modelo Tobit, devido a variável dependente ser censurada (de 0 a 100). Foram utilizados controle de efeito fixo por setor e ano, além de erros padrão robustos. A operacionalização foi realizada no software Stata versão 13. A equação geral utilizada é a seguinte:

$R S C i t=\beta 0+\beta 1$ INTERit $+\beta 2$ TAMit $+\beta 3$ GC_Gest $+\beta 4$ CG_Aciit $+\beta 5$ ROAit + EEfeitos Fixos_SETOR + EEfeitos Fixos_ANO $+\varepsilon$

A variável setor, refere-se à classificação contida na Thomson Reuters Eikon, classificada pela nomenclatura "NAICS Sector Name", a qual se refere aos setores de atuação das empresas da amostra. Semelhantemente, a variável ano refere-se aos anos de 2007 a 2017.

Os pressupostos dos modelos de regressão linear múltipla (homocedasticidade, multicolinearidade, autocorrelação dos resíduos e normalidade) foram testados antes de se proceder a análise dos dados, assim como a estatística descritiva e o teste de correlação entre as variáveis. $O$ problema de heterocedasticidade foi solucionado com a utilização de erros padrões robustos. O pressuposto da normalidade pode ser relaxado em função da quantidade de observações. Os testes VIF (multicolinearidade) e Durbin-Watson (autocorrelação dos resíduos) foram apresentados nas tabelas dos resultados.

\section{APRESENTAÇÃO E DISCUSSÃO DOS RESULTADOS}

A análise inicial apresenta a estatística descritiva das variáveis do estudo e o teste t de Student, utilizado para verificar se existem diferenças significativas entre as variáveis de empresas de diferentes grupos (reguladas versus não reguladas e com ADR versus sem ADR).

Tabela 3 - Resultados da análise descritiva

\begin{tabular}{|c|c|c|c|c|c|c|c|}
\hline \multirow{2}{*}{ Variáveis } & Amostra Total & Reguladas & NR & \multirow{2}{*}{ Teste $\mathrm{t}$} & Com ADR & Sem ADR & \multirow{2}{*}{ Teste $\mathrm{t}$} \\
\hline & Média & Média & Média & & Média & Média & \\
\hline \multirow[t]{2}{*}{ RSC } & 57,36 & 63,69 & 54,57 & $5,15^{*}$ & 68,10 & 52,87 & $9,00^{*}$ \\
\hline & $(18,76)$ & $(18,41)$ & $(18,26)$ & & $(12,17)$ & $(19,22)$ & \\
\hline \multirow[t]{2}{*}{ RSC_Soc } & 61,09 & 68,90 & 57,65 & $5,59^{* *}$ & 71,87 & 56,60 & $7,81^{*}$ \\
\hline & $(21,65)$ & $(19,70)$ & $(21,60)$ & & $(14,80)$ & $(22,47)$ & \\
\hline \multirow[t]{2}{*}{ RSC_Amb } & 53,62 & 58,47 & 51,49 & $3,41^{*}$ & 64,34 & 49,15 & $7,91^{*}$ \\
\hline & $(20,61)$ & $(21,37)$ & $(19,92)$ & & $(15,67)$ & $(20,79)$ & \\
\hline \multirow[t]{2}{*}{ INTER } & 0,03 & 0,00 & 0,04 & $-4,67^{*}$ & 0,052 & 0,02 & $3,33^{*}$ \\
\hline & $(0,08)$ & $(0,02)$ & $(0,10)$ & & $(0,09)$ & $(0,08)$ & \\
\hline \multirow[t]{2}{*}{ TAM } & 22,48 & 23,07 & 22,22 & $7,15^{*}$ & 23,56 & 22,02 & $15,46^{*}$ \\
\hline & $(1,25)$ & $(1,25)$ & $(1,16)$ & & $(1,08)$ & $(1,01)$ & \\
\hline GC_Ges & 50,70 & 45,49 & 52,99 & $-2,88^{*}$ & 56,98 & 48,08 & $3,34^{*}$ \\
\hline
\end{tabular}




\begin{tabular}{|c|c|c|c|c|c|c|c|}
\hline & $(27,29)$ & $(26,73)$ & $(27,26)$ & & $(27,58)$ & $(26,78)$ & \\
\hline \multirow[t]{2}{*}{ GC_Aci } & 52,18 & 50,42 & 52,95 & $-1,04$ & 39,72 & 57,38 & $-6,69^{*}$ \\
\hline & $(29,21)$ & $(28,17)$ & $(29,66)$ & & $(26,26)$ & $(28,84)$ & \\
\hline \multirow[t]{2}{*}{ ROA } & 0,07 & 0,07 & 0,08 & $-0,87^{*}$ & 0,08 & 0,07 & $0,13^{*}$ \\
\hline & $(0,07)$ & $(0,06)$ & $(0,07)$ & & $(0,05)$ & $(0,07)$ & \\
\hline \multirow{2}{*}{ Observações } & \multirow{2}{*}{530} & 162 & 368 & & 156 & 374 & \\
\hline & & \multicolumn{2}{|c|}{530} & & \multicolumn{2}{|c|}{530} & \\
\hline
\end{tabular}

Nota: *Significância ao nível de 1\%. **Significância ao nível de 5\%. ** Significância ao nível de 10\%. Amostra Total: refere-se a todas as empresas da amostra. Reguladas: refere-se apenas as empresas dos setores regulados. NR: refere-se as empresas pertencentes aos setores não regulados. Com ADR: refere-se às empresas que estão listadas na NYSE. Sem ADR: refere-se às empresas que não estão listadas na NYSE. Valores entre parênteses: desvio padrão.

Fonte: Dados da pesquisa (2018)

Em relação à amostra total, destaca-se que o valor médio de $\operatorname{RSC}(57,36)$ representa que as empresas atendem a mais da metade dos critérios de RSC e que os critérios mais evidenciados são referentes à dimensão social. Com relação à internacionalização, observa-se que em média as empresas possuem $3,7 \%$ de seus ativos, receita e empregados no exterior, percentual que se demonstra consideravelmente disperso entre as empresas, o que pode ser observado pelo desvio padrão.

No que se refere às empresas reguladas e não reguladas, observa-se que em média as primeiras divulgam 15\% mais informações sobre questões sociais e ambientais do que as não reguladas. Este resultado pode ser atribuído ao fato de que as empresas reguladas são regidas por um órgão que delimita obrigatoriamente certas responsabilidades. Além disso, ao analisar a média de internacionalização, percebe-se que empresas reguladas são estatisticamente menos internacionalizadas do que as demais, o que gera indícios preliminares de que não necessariamente a internacionalização impacta nas práticas de RSC para esse grupo de empresas.

A separação entre empresas que possuem e não ADR's foi realizada no intuito de observar se as empresas expostas a demanda dos shareholders estrangeiros possuem maior divulgação de informações sobre RSC. Assim, percebeu-se que as empresas com ADR's possuem média de RSC 22\% superior as demais, o que revela que esse grupo de empresas possui incentivos para se engajar em práticas de RSC. Por fim, ao observar os valores de internacionalização, observa-se que as empresas com ADR's possuem em média maior índice de internacionalização.

Ressalta-se que o teste $t$ de Student demonstrou valores de RSC (ambiental, social e a dimensão geral) estatisticamente diferentes entre os grupos de empresas reguladas e não reguladas, assim como a variável tamanho, GC_Ges e o ROA, com exceção da variável de GC_Aci. Com relação aos resultados do teste $t$ de Student para os grupos de empresas com e sem ADR, observou-se que todas as variáveis se mostraram estatisticamente diferentes.

\subsection{Análise da relação entre internacionalização e RSC}

Na Tabela 4, apresentam-se os resultados da influência da internacionalização nas práticas de RSC. Para esta análise, observam-se os resultados da Equação 1.

Tabela 4 - Resultados da influência da internacionalização nas práticas de RSC

\begin{tabular}{|c|c|c|c|}
\hline \multirow{2}{*}{ Variáveis } & \multicolumn{3}{|c|}{ RSC } \\
\cline { 2 - 4 } & Coeficiente & $\mathrm{t}$ & VIF \\
\hline Constante & $-158,777^{*}$ & $-11,34$ & 1,40 \\
\hline INTER & $22,24324^{*}$ & 3,01 & 1,64 \\
\hline TAM & $8,623431^{*}$ & 15,83 & 1,19 \\
\hline GC_Ges & $0,1643888^{*}$ & 6,66 & \\
\hline
\end{tabular}




\begin{tabular}{|c|c|c|c|}
\hline GC_Aci & $0,0530564^{* *}$ & 2,18 & 1,42 \\
\hline ROA & $57,23093^{*}$ & 6,08 & 1,30 \\
\hline Efeito fixo setor & \multicolumn{3}{|c|}{ Sim } \\
\hline Efeito fixo ano & \multicolumn{3}{|c|}{ Sim } \\
\hline $\mathrm{R}^{2}$ & \multicolumn{3}{|c|}{0,0724} \\
\hline Significância & \multicolumn{3}{|c|}{$0,0000^{*}$} \\
\hline Durbin Watson & \multicolumn{3}{|c|}{1,877899} \\
\hline Observaçōes & \multicolumn{3}{|c|}{530} \\
\hline
\end{tabular}

Com base na Tabela 4, percebe-se que o modelo foi significativo, com $R^{2}$ de 7,24\%. Além disso, observa-se que as variáveis não estão expostas a graves problemas de multicolinearidade (VIF), nem de autocorrelação dos resíduos (Durbin Watson).

O grau de internacionalização se mostrou positivamente relacionado à RSC, isso sugere que as empresas com parte do ativo, receita e/ou empregados no exterior, realizam e divulgam mais informações sobre seu comportamento socialmente responsável. Tal comportamento pode ser explicado pela teoria dos stakeholders de Freeman (1994), a qual afirma que as práticas de RSC são realizadas com a intenção de gerar uma contrapartida às partes afetadas pelas atividades das empresas, como a sociedade, os funcionários, os investidores, meio ambiente, entre outros. Dessa forma, depreende-se que as empresas internacionalizadas estão expostas à dualidade de stakeholders e, como consequência disso, recebem maior demanda por informações sobre seu comportamento e passam a investir e divulgar suas ações de RSC.

Com relação aos resultados empíricos, destaca-se que pesquisas anteriores realizadas em outros países, como a de Brammer et al. (2009), Kang (2013), Attig et al. (2016), Hadjikhani et al. (2016) e de Agnihotri e Bhattacharya (2016), evidenciaram resultados condizentes aos constatados nesta pesquisa. Portanto, os achados deste estudo sugerem uma evidência para o cenário brasileiro, onde assim como em outros contextos, as empresas que se expandem internacionalmente, por estarem expostas a maior pressão dos stakeholders, se veem em posição de melhorar suas práticas de RSC, como forma de se inserir ou se manter no mercado estrangeiro.

Além da motivação oriunda da pressão de dois grupos de stakeholders (do país de origem e de acolhimento), o engajamento em RSC pode ser percebido como uma estratégia de expansão das organizações, que buscam expandir suas atividades no exterior já com um diferencial competitivo (Bondy \& Starkey, 2014).

Quanto às variáveis de controle, nota-se que empresas maiores estão relacionadas positivamente a maiores scores de RSC, assim como o proposto por Attig et al. (2016), os quais sustentam que tais empresas têm maior capacidade de lidar com as incertezas de operações no exterior e, portanto, se adaptam melhor às práticas de RSC exigidas no processo de internacionalização.

Observou-se ainda que os mecanismos de GC, tanto os direcionados ao alinhamento de interesse da gestão da empresa (GC_Ges), como dos acionistas (GC_Aci), influenciam o comportamento socialmente responsável das empresas. Este resultado coaduna com o exposto por Ali et al. (2017), de que empresas que possuem administradores não executivos no conselho ou que possuem comitê de auditoria influenciam positivamente a divulgação de RSC.

Ainda de acordo com Ali et al. (2017), tem-se que empresas com elevados índices de rentabilidade são mais propensas a investirem em ações de RSC, o que foi comprovado em empresas brasileiras, onde 
a medida de rentabilidade sobre o ativo se mostrou positivamente relacionada à RSC. Essa evidência infere sobre o fato de que empresas maiores e mais rentáveis são mais visíveis e, portanto, mais pressionadas pelos seus stakeholders com relação à exigência de práticas socialmente responsáveis e além disso, possuem mais recursos, de modo a facilitar o engajamento em RSC.

$\mathrm{Na}$ Tabela 5 estão expostos os resultados sobre a influência da internacionalização nas dimensões social e ambiental da RSC. Esta análise foi realizada com a intenção de verificar se a internacionalização influencia igualmente as diferentes dimensões de RSC.

Tabela 5 - Resultados da influência da internacionalização nas dimensões de RSC

\begin{tabular}{|c|c|c|c|c|c|c|}
\hline \multirow{2}{*}{ Variáveis } & \multicolumn{3}{|c|}{ RSC_Soc } & \multicolumn{3}{|c|}{ RSC_Amb } \\
\hline & Coeficiente & $\mathrm{t}$ & VIF & Coeficiente & $\mathrm{t}$ & VIF \\
\hline Constante & $-159,3546^{*}$ & $-11,16$ & & $-156,826^{*}$ & $-7,68$ & \\
\hline INTER & $37,74474^{*}$ & 5,04 & 1,40 & 6,280281 & 0,61 & 1,40 \\
\hline TAM & $9,077816^{*}$ & 14,98 & 1,64 & $8,117725^{*}$ & 11,36 & 1,64 \\
\hline GC_Ges & $0,242227^{*}$ & 8,80 & 1,19 & $0,0863337^{*}$ & 2,85 & 1,19 \\
\hline GC_Aci & $0,0769041^{* *}$ & 2,56 & 1,42 & 0,0287564 & 1,02 & 1,42 \\
\hline ROA & $42,57469^{*}$ & 3,85 & 1,30 & $71,10488^{*}$ & 6,31 & 1,30 \\
\hline Efeito fixo setor & \multicolumn{2}{|c|}{ Sim } & & \multicolumn{2}{|c|}{ Sim } & \\
\hline Efeito fixo ano & \multicolumn{2}{|c|}{ Sim } & & \multicolumn{2}{|c|}{ Sim } & \\
\hline $\mathrm{R}^{2}$ & \multicolumn{2}{|c|}{0,0731} & & \multicolumn{2}{|c|}{0,0473} & \\
\hline Significância & \multicolumn{2}{|c|}{$0,0000^{*}$} & & \multicolumn{2}{|c|}{$0,0000^{*}$} & \\
\hline Durbin Watson & \multicolumn{2}{|c|}{1,879952} & & \multicolumn{2}{|c|}{2,095379} & \\
\hline Observações & \multicolumn{2}{|c|}{530} & & \multicolumn{2}{|c|}{530} & \\
\hline
\end{tabular}

Nota: *Significância ao nível de 1\%. **Significância ao nível de 5\%. *** Significância ao nível de 10\%. VIF: Variance Inflation Factor. Fonte: Dados da pesquisa (2018)

Os resultados da Tabela 5 apontam que a internacionalização se mostrou estatisticamente significante apenas para as práticas de RSC direcionadas à dimensão social (RSC_Soc), isso permite inferir que o fato da empresa se expandir internacionalmente faz com que a mesma invista em aspectos relacionados à responsabilidade com a comunidade, com os direitos humanos e com a força de trabalho.

Os itens que compõem a dimensão social da RSC são relacionados à questões voltadas à prática contra suborno e corrupção, à políticas que visam a ética empresarial, ao envolvimento da comunidade, a saúde e segurança dos empregados, as iniciativas de comércio ético, entre outros.

Attig et al. (2016) atentam para o fato de que os resultados sobre dimensões individuais de RSC devem ser analisados com cautela, pois é difícil avaliar até que ponto os stakeholders conseguem analisar as dimensões de RSC separadamente, como por exemplo, ações sociais e ambientais testadas neste estudo.

Todavia, existem na literatura algumas justificativas a serem consideradas para auxiliar no entendimento sobre o porquê determinadas práticas de RSC são influenciadas pela internacionalização e outras não. Brammer et al. (2009) investigaram a influência da internacionalização nas doações corporativas em empresas do Reino Unido, entretanto, por mais que a pesquisa se destinou a um país específico, gerou evidências com relação às diferenças geográficas de países chamados pelos autores de "preocupantes". Portanto, os autores analisaram o Reino Unido especificamente, mas lançaram luz sobre as possíveis diferenças que podem influenciar a relação entre RSC e internacionalização.

Brammer et al. (2009) apoiam a visão de que os stakeholders relacionam as empresas aos países em que estão localizadas e aos comportamentos políticos, sociais, corporativos ou ambientais deste país. Os autores exemplificam essa questão citando países fictícios os quais podem estar associados ao abuso de direitos humanos, à falta de liberdade política ou à corrupção política/corporativa. Assim, os stakeholders 
percebem um risco de associação das empresas com as características negativas daquele país. Portanto, as organizações podem sofrer maior pressão dos stakeholders sobre certas dimensões de RSC, pois estas se relacionam às questões controversas que cercam as operações das empresas multinacionais.

No Brasil, de acordo com o relatório publicado pela organização independente Freedom House, em 2017, as dificuldades enfrentadas no país se relacionavam com a corrupção, o crime e a exclusão econômica das minorias. Além disso, de acordo com o site do Transparency International, o Brasil foi classificado em 40 pontos, no relatório de 2017 (quanto mais próximo de 0 mais corrupto é o país) e está em $96^{\circ}$ colocação de 180 empresas, o que s que o país está localizado no terceiro quartil de empresas e, portanto, se mostra mais próximo dos países com altos índices de corrupção.

Com base nisto, observa-se que as controversas as quais cercam as empresas brasileiras são relacionadas às questões sociais e não ambientais. Talvez, em decorrência disso, as empresas divulgam mais informações sociais com o intuito de se mostrarem desassociadas das questões negativas do país aos seus stakeholders, para que assim consigam se estabelecer ou manter suas atividades no exterior.

\subsection{Testes de sensibilidade/robustez}

Com a intenção de melhor explorar os resultados, separou-se as empresas em reguladas e não reguladas, a fim controlar essa característica da amostra e assegurar que os resultados obtidos na análise principal não são enviesados pelas empresas reguladas. Os resultados deste teste estão expostos na Tabela 6.

Tabela 6 - Resultados da regressão em empresas reguladas e não reguladas

\begin{tabular}{|c|c|c|c|c|c|c|}
\hline \multirow{2}{*}{ Variáveis } & \multicolumn{2}{|c|}{ RSC - Empresas Reguladas } & \multicolumn{2}{|c|}{ RSC - Empresas Não Reguladas } & \multicolumn{2}{|c|}{ RSC } \\
\cline { 2 - 7 } & Coeficiente & VIF & Coeficiente & VIF & Coeficiente & VIF \\
\hline Constante & $-214,0442^{*}$ & & $-157,663^{*}$ & & $-128,03^{*}$ & \\
\hline & $(-5,37)$ & & $(-8,82)$ & & $(-9,02)$ & \\
\hline INTER & 61,4167 & 4,65 & $18,13288^{* *}$ & 1,47 & 9,62939 & 1,21 \\
\hline & $(1,19)$ & & $(2,48)$ & & $(1,29)$ & \\
\hline REGUL & & & & & $3,840868^{* *}$ & 1,13 \\
\hline & & & & & $(2,50)$ & \\
\hline TAM & $11,4124^{*}$ & 3,51 & $8,368883^{*}$ & 2,05 & $8,147318^{*}$ & 1,29 \\
\hline & $(7,08)$ & & $(10,34)$ & & $(15,03)$ & \\
\hline GC_Ges & $0,2350014^{*}$ & 1,33 & $0,1358625^{*}$ & 1,19 & $0,1433063^{*}$ & 1,03 \\
\hline & $(4,81)$ & & $(4,92)$ & & $(6,35)$ & \\
\hline GC_Aci & $-0,0672418^{* * *}$ & 1,53 & $0,1185688^{*}$ & 1,53 & 0,0092172 & 1,08 \\
\hline & $(-1,72)$ & & $(3,76)$ & & $(0,40)$ & \\
\hline ROA & $71,72296^{*}$ & 1,51 & $51,26995^{*}$ & 1,45 & $47,48551^{*}$ & 1,09 \\
\hline & $(3,45)$ & & $(4,37)$ & & $(5,24)$ & \\
\hline Efeito fixo setor & Sim & & Sim & & Não & \\
\hline Efeito fixo ano & Sim & & Sim & & Sim & \\
\hline R & 0,0881 & & 0,0729 & & 0,0554 & \\
\hline Significância & $0,0000^{*}$ & & $0,0000^{*}$ & & $0,0000^{*}$ & \\
\hline Durbin Watson & 2,431866 & & 1,843498 & & 1,902079 & \\
\hline Observaçöes & 162 & & 368 & & 530 & \\
\hline
\end{tabular}

Nota: *Significância ao nível de 1\%. **Significância ao nível de 5\%. *** Significância ao nível de 10\%. VIF: Variance Inflation Factor. Valores entre parênteses: estatística t.

Fonte: Dados da pesquisa (2018) 
Nota-se na Tabela 6 que a internacionalização se demonstrou estatisticamente significante apenas para o grupo de empresas não reguladas, isso sugere que para o grupo de empresas reguladas, a internacionalização não tem efeito nas práticas de RSC. Tais resultados demonstram que essas empresas se engajam em práticas de RSC motivadas pela regulamentação e não pelo fato de serem internacionalizadas.

Para confirmar essa percepção, operacionalizou-se uma terceira regressão (terceira coluna da Tabela 6), a qual considerou a regulação como uma variável dicotômica (1 para empresas reguladas, 0 caso contrário). Os resultados demonstraram que a variável regulação (REGUL) se mostrou positivamente relacionada às práticas de RSC, enquanto que a variável internacionalização (INTER) não demonstrou significância estatística. Essas evidências confirmam que as práticas de RSC realizadas pelas empresas reguladas são motivadas pelo fator regulatório de seus setores e não pela internacionalização.

A motivação para as empresas reguladas se comportarem de forma socialmente responsável foi destacada conceitualmente desde o estudo seminal de Carroll (1979). Portanto, ao analisar RSC, é importante controlar os fatores regulatórios relacionados as peculiaridades de cada setor, visto que nesta pesquisa a RSC se mostrou motivada pela regulação, e não pela internacionalização das empresas.

Na Tabela 7 estão expostos os resultados da análise da relação entre a RSC e a internacionalização moderada pela variável ADR, que representa as empresas brasileiras com ações negociadas na NYSE.

\begin{tabular}{|c|c|c|c|}
\hline \multirow{2}{*}{ Variáveis } & \multicolumn{3}{|c|}{ RSC } \\
\hline & Coeficiente & $\mathrm{t}$ & VIF \\
\hline Constante & $-156,1994$ & $-9,47$ & \\
\hline INTER & $18,72681^{* *}$ & 1,99 & 2,03 \\
\hline ADR & $-0,2034403$ & $-0,11$ & 2,10 \\
\hline INTER*ADR & 11,16163 & 0,73 & 2,77 \\
\hline TAM & $8,474256^{*}$ & 11,86 & 2,36 \\
\hline GC_Ges & $0,1634651^{*}$ & 6,30 & 1,28 \\
\hline GC_Aci & $0,05636^{* *}$ & 2,22 & 1,51 \\
\hline ROA & $56,16882^{*}$ & 5,78 & 1,35 \\
\hline Efeito fixo setor & \multicolumn{2}{|c|}{ Sim } & \\
\hline Efeito fixo ano & \multicolumn{2}{|c|}{ Sim } & \\
\hline$R^{2}$ & \multicolumn{2}{|c|}{0,0725} & \\
\hline Significância do modelo & \multicolumn{2}{|c|}{$0,0000^{*}$} & \\
\hline Durbin Watson & \multicolumn{2}{|c|}{1,876493} & \\
\hline Observações & \multicolumn{2}{|c|}{530} & \\
\hline
\end{tabular}

Nota: *Significância ao nível de 1\%. **Significância ao nível de 5\%. ** Significância ao nível de 10\%. VIF: Variance Inflation Factor. Fonte: Dados da pesquisa (2018)

Com base na Tabela 7, nota-se que as variáveis ADR e sua interação com a internacionalização (INTER*ADR) não se mostraram significantes, o que reafirma o pressuposto teórico desta pesquisa de que a internacionalização, mensurada pelo nível de ativo, empregados e receita no exterior, é um determinante para o engajamento em RSC, ao contrário da expansão no exterior mediante listagem na NYSE.

Por fim, com a intenção de verificar se a constância de algumas empresas na amostra nos primeiros anos não poderia enviesar os resultados, realizou-se a mesma regressão da análise principal, com a exclusão dos 3 primeiros anos (2007, 2008 e 2009). Os resultados estão expostos na Tabela 8. 
Tabela 8 - Resultados do modelo principal no período de 2010 a 2017

\begin{tabular}{|c|c|c|c|}
\hline \multirow{2}{*}{ Variáveis } & \multicolumn{3}{|c|}{ RSC } \\
\hline & Coeficiente & $\mathrm{t}$ & VIF \\
\hline Constante & $-159,15^{*}$ & $-13,28$ & \\
\hline INTER & $22,29511^{*}$ & 2,90 & 1,36 \\
\hline TAM & $8,723508^{*}$ & 15,86 & 1,61 \\
\hline GC_Ges & $0,1667606^{*}$ & 6,44 & 1,20 \\
\hline GC_Aci & $0,0522961^{* *}$ & 2,07 & 1,48 \\
\hline ROA & $55,79929^{*}$ & 5,84 & 1,29 \\
\hline Efeito fixo setor & \multicolumn{2}{|c|}{ Sim } & \\
\hline Efeito fixo ano & \multicolumn{2}{|c|}{ Sim } & \\
\hline$R^{2}$ & \multicolumn{2}{|c|}{0,0738} & \\
\hline Significância do modelo & \multicolumn{2}{|c|}{$0,0000^{*}$} & \\
\hline Durbin Watson & \multicolumn{2}{|c|}{2,290503} & \\
\hline Observações & \multicolumn{2}{|c|}{494} & \\
\hline
\end{tabular}

Nota: *Significância ao nível de 1\%. **Significância ao nível de 5\%. *** Significância ao nível de 10\%. VIF: Variance Inflation Factor. Fonte: Dados da pesquisa (2018)

Observa-se na Tabela 8 que as variáveis mantiveram seus resultados. Portanto, pode-se inferir que a repetição de empresas nos três primeiros anos não enviesou os resultados. De forma geral, observou-se que após os testes de sensibilidade, os resultados demonstraram que a internacionalização se mostrou positivamente relacionada com as práticas de RSC, com exceção para as empresas reguladas. Portanto, pode-se inferir a robustez nos resultados e diante disso, não se rejeita a $\mathrm{H} 1$. Contudo, atenta-se para o fato de que a internacionalização é um fator determinante do engajamento em RSC, apenas para as empresas não pertencentes à setores regulados.

\section{CONCLUSÕES}

A presente investigação analisou a influência da internacionalização nas práticas de RSC em 71 empresas brasileiras, no período de 2007 a 2017. Os resultados evidenciaram que a internacionalização apresenta uma relação positiva com as práticas de RSC. Contudo, ao analisar a RSC por suas dimensões (social e ambiental), notou-se que apenas as práticas voltadas à comunidade, aos direitos humanos e a força de trabalho (dimensão social) são influenciadas pela internacionalização.

A justificativa para estes resultados vem de Brammer et al. (2009), que explanam sobre a associação das empresas às questões controversas de seu país de origem, percebido pelos stakeholders como um risco de associação. O Brasil enfrenta dificuldades associadas à corrupção, crise e exclusão econômica das minorias (Freedom House, 2017), questões estas que dizem respeito a aspectos sociais e não ambientais, o que poderia, de certa forma, explicar tal resultado.

Com relação aos demais resultados, depreende-se que a internacionalização influencia positivamente a RSC apenas para as empresas não reguladas. As empresas cujos setores são regulados possuem maior comprometimento com os níveis de RSC, isso decorre do fator regulatório e não da expansão internacional das organizações. Portanto, ao analisar práticas de RSC, torna-se relevante controlar fatores regulatórios dos setores, visto que estes se mostram como determinantes para a RSC.

Em comparação aos estudos concentrados na América do Norte e Europa, nota-se que estes achados vão ao encontro dos obtidos por Brammer et al. (2009) no Reino Unido, Attig et al. (2016) na América do Norte, Hadjikhani et al. (2016) na Coréia e China e Agnihotri e Bhattacharya (2016) na Índia. Dessa forma, confirmam a relação positiva entre RSC e internacionalização no cenário brasileiro. Por outro 
lado, os achados divergem dos evidenciados por Bondy e Starkey (2014) no Reino Unido e Aguilera-Caracuel et al. (2014) nos EUA.

As evidências encontradas contribuem com a literatura sobre RSC, principalmente a relacionada aos seus determinantes, pois destaca que não só preocupações sociais e ambientais sustentam tais práticas, mas decisões estratégicas relacionadas à inserção no mercado externo também podem influenciar tal comportamento. De forma prática, as contribuições estão voltadas à inclusão de pesquisa empírica no Brasil, por ser tratar de um país emergente e em função de que as características dos países podem influenciar no engajamento das organizações em práticas de RSC.

Em relação aos estudos anteriores, esta pesquisa avançou no sentido de explorar as diferenças na influência da internacionalização nas práticas de RSC para empresas de setores regulados e não regulados, o que gerou inclusive resultados adicionais à literatura. Além disso, avançou por abordar uma medida de RSC que considera aspectos quantitativos e qualitativos, os quais são passíveis de serem investigados por seus aspectos ambientais e/ou sociais.

Contudo, este estudo apresenta limitações. Não foi considerada a cultura nacional dos países onde as subsidiárias estão localizadas, o que para Aguilera-Caracuel et al. (2014) pode influenciar as empresas a se engajarem em RSC no processo de internacionalização. Além disso, o número reduzido de empresas brasileiras que divulgam suas práticas de RSC se mostrou uma limitação, por restringir a amostra em 71 empresas que possuem estas informações. Portanto, sugere-se para pesquisas futuras investigar a influência da internacionalização na RSC em outros contextos e inserir nesta análise características da cultura nacional e da situação econômica do país de origem e acolhimento.

\section{REFERÊNCIAS}

Abreu, M. C. S., Castro, F., de Assis Soares, F., \& da Silva Filho, J. C. L. (2012). A comparative understanding of corporate social responsibility of textile firms in Brazil and China. Journal of Cleaner Production, 20(1), 119-126. https://doi.org/10.1016/j.jclepro.2011.08.010

Agnihotri, A., \& Bhattacharya, S. (2016). Communicating CSR practices-Role of internationalization of emerging market firms. Journal of Marketing Communications, 22(1), 1-20. https://doi.org/10.1080/1 3527266.2016.1251488

Aguilera-Caracuel, J., Delgado-Márquez, B. L., \& Vidal-Salazar, M. D. (2014). Influencia de la internacionalización en el desempeño social de las empresas/The influence of internationalization on firm corporate social performance. Cuadernos de Gestión, 14(2), 15-32. https:// 10.5295/cdg.130420ja

Ali, W., Frynas, J. G., \& Mahmood, Z. (2017). Determinants of corporate social responsibility (CSR) disclosure in developed and developing countries: A literature review. Corporate Social Responsibility and Environmental Management, 24(4), 273-294. https://doi/pdf/10.1002/csr.1410

Attig, N., Boubakri, N., El Ghoul, S., \& Guedhami, O. (2016). Firm internationalization and corporate social responsibility. Journal of Business Ethics, 134(2), 171-197. https://doi.10.1007/s10551-014-2410-6

Bondy, K., \& Starkey, K. (2014). The dilemmas of internationalization: Corporate social responsibility in the multinational corporation. British Journal of Management, 25(1), 4-22. https://doi.org/10.1111/j. 1467-8551.2012.00840.x

Brammer, S. J., Pavelin, S., \& Porter, L. A. (2009). Corporate charitable giving, multinational companies and countries of concern. Journal of Management Studies, 46(4), 575-596. https://doi.org/10.1111/j. 1467-6486.2008.00827.x

Campbell, J. T., Eden, L., \& Miller, S. R. (2012). Multinationals and corporate social responsibility in host countries: Does distance matter?. Journal of International Business Studies, 43(1), 84-106. 
Carroll, A. B. (1979). A three-dimensional conceptual model of corporate performance. Academy of Management Review, 4(4), 497-505. https://doi.org/10.5465/amr.1979.4498296

Chapple, W., \& Moon, J. (2005). Corporate social responsibility (CSR) in Asia: A seven-country study of CSR web site reporting. Business \& Society, 44(4), 415-441. https://doi.org/10.1016/j.ibusrev.2009.12.003

Choi, M. (2017). The efffect of corporate social responsibility performance on internationalization strategy and firm value. Journal of International Trade \& Commerce, 13(2), 443-457. http://dx.doi.org/10.16980/ jitc.13.2.201704.443

Detomasi, D. A. (2008). The political roots of corporate social responsibility. Journal of Business Ethics, 82(4), 807-819. https://doi. 10.1007/s10551-007-9594-y

Freedom House. Relatório de País. Recuperado em 15 fevereiro, 2018, de https://freedomhouse.org/ report/freedom-world/2017/brazil.

Freeman, R. E. (1994). The politics of stakeholder theory: Some future directions. Business Ethics Quarterly, 4(4), 409-421. https://doi. 10.2307/3857340

Gallego-Álvarez, I., \& Ortas, E. (2017). Corporate environmental sustainability reporting in the context of national cultures: A quantile regression approach. International Business Review, 26(2), 337-353. https://doi.org/10.1016/j.ibusrev.2016.09.003

Gulamhussen, M. A., Pinheiro, C., \& Pozzolo, A. F. (2014). International diversification and risk of multinational banks: Evidence from the pre-crisis period. Journal of Financial Stability, 13, 30-43. https://doi. org/10.1016/j.jfs.2014.02.007

Hadjikhani, A., Lee, J. W., \& Park, S. (2016). Corporate social responsibility as a marketing strategy in foreign markets: the case of Korean MNCs in the Chinese electronics market. International Marketing Review, 33(4), 530-554. https://doi.org/10.1108/IMR-03-2014-0104

Jung, S., Lee, S., \& Dalbor, M. (2016). The negative synergistic effect of internationalization and corporate social responsibility on US restaurant firms' value performance. International Journal of Contemporary Hospitality Management, 28(8), 1759-1777. https://doi.org/10.1108/IJCHM-07-2014-0361

Kang, J. (2013). The relationship between corporate diversification and corporate social performance. Strategic Management Journal, 34(1), 94-109. https://doi.org/10.1002/smj.2005

Kolk, A., \&Van Tulder, R. (2010). International business, corporate social responsibility and sustainable development. International business review, 19(2), 119-125. https://doi.org/10.1016/j.ibusrev.2009.12.003

Kim, Y., \& Kim, S. Y. (2010). The influence of cultural values on perceptions of corporate social responsibility: Application of Hofstede's dimensions to Korean public relations practitioners. Journal of Business Ethics, 91(4), 485-500. https://doi. 10.1007/s10551-009-0095-z

Mazzioni, S. (2015). Influência do grau de internacionalização das empresas na relação entre as normas reguladoras e os incentivos empresariais com a qualidade das informações contábeis. Tese de doutorado. Universidade Regional de Blumenau, Blumenau, SC, Brasil.

Orlitzky, M., Siegel, D. S., \&Waldman, D. A. (2011). Strategic corporate social responsibility and environmental sustainability. Business \& Society, 50(1), 6-27. https://doi.org/10.1177\%2F0007650310394323

Serra, F. A. R., Albernaz, A., \& Ferreira, M. P. (2007). A Responsabilidade social como fator na estratégia internacional: o estudo do caso natura. Revista Eletrônica de Administração, 13(4), 17-39.

Shnayder, L., Van Rijnsoever, F. J., \& Hekkert, M. P. (2016). Motivations for corporate social responsibility in the packaged food industry: an institutional and stakeholder management perspective. Journal of Cleaner Production, 122, 212-227. https://doi.org/10.1016/j.jclepro.2016.02.030

Transparency International. Índice de percepção de corrupção 2016. Recuperado em 15 fevereiro, 2018, de https://www.transparency.org/country/BRA.

Zahra, S. A., Ireland, R. D., \& Hitt, M. A. (2000). International expansion by new venture firms: International diversity, mode of market entry, technological learning, and performance. Academy of Management Journal, 43(5), 925-950. https://doi.org/10.5465/1556420 